\title{
Secondary electron emission and photoemission studies on surface films of carbon nitride
}

\author{
J. M. Ripalda \\ Instituto de Microelectrónica de Madrid, CSIC, PTM, Tres Cantos, Madrid 28760, Spain \\ I. Montero ${ }^{\text {a) }}$ and L. Vázquez \\ Instituto de Ciencia de Materiales de Madrid, CSIC, Cantoblanco, Madrid 28049, Spain \\ D. Raboso \\ European Space Research and Technology Center, ESA, 2200 AG Noordwijk, Holland \\ L. Galán \\ Departamento de Física Aplicada, Universidad Autónoma de Madrid, Cantoblanco, Madrid 28049, Spain
}

(Received 8 April 2005; accepted 16 January 2006; published online 24 February 2006)

\begin{abstract}
The secondary electron emission yield of fullerene, graphite, and diamondlike carbon after low-energy $\mathrm{N}_{2}{ }^{+}$ion bombardment was studied for antimultipactor applications. Nitrogen incorporation into the carbon thin films decreases their secondary emission yield, contrary to the hydrogen or oxygen effect. Carbon nitride surface textured to a nanometric scale had the property of hindering secondary electron emission. Valence bands obtained from photoemission spectroscopy using synchrotron radiation were correlated with secondary electron emission measurements. Multipactor threshold power for carbon nitride was $7.5 \mathrm{~kW}$. () 2006 American Institute of Physics. [DOI: $10.1063 / 1.2173307]$
\end{abstract}

\section{INTRODUCTION}

The multipactor effect is a resonant radio-frequency (rf) electron discharge in vacuum sustained by secondary electron emission from the walls of radio-frequency devices. ${ }^{1-7}$ Multipactor discharge is caused by electron multiplication due to emission of secondary electrons from surface materials, due to the impact of rf energized electrons. It is an important problem in high-power rf devices in vacuum, such as telecommunication and remote sensing equipment in satellites, rf cavities in particle accelerators, and rf plasma heating devices in nuclear fusion experiments. ${ }^{3-7}$ Usually, several conditions have to be fulfilled for the apparition of the multipactor effect, namely, (a) a phase resonance must exist, (b) primary electrons accelerated by the rf field must have an energy such that the secondary electron emission yield of the impacted surface must exceed unity, and (c) the product of operating frequency by gap spacing $(f d)$ must fall within a range which ensures that the electron transit time is in the order of an odd multiple of one-half rf cycle.

The search for reliable low secondary electron emission materials, which would reduce the feedback necessary for the electron avalanche, is an important technological issue. The main objective of this research is to find a stable and inert surface with respect to secondary electron emission properties, or, at least, one that evolves favorably under the initial stages of multipactor discharge, i.e., upon low-energy electron and ion bombardment. In this work we present results about the relationship between the secondary electron emission and multipactor properties. The materials studied were fullerene, diamondlike carbon, and graphite before and

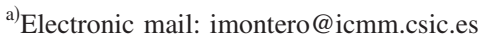

after the nitridation process. Surface physics spectroscopic and microscopic techniques were used in combination with secondary electron emission measurements.

Oblique incidence angles imply a longer part of the penetration range of the primary electron being inside a nearsurface region from which secondaries can escape from, therefore leading to higher secondary electron emission yield (SEY). However, there are also shadowing effects of the protruding surface structures over the concave zones that hamper their SEY. These effects are different for emitted true secondary electrons (low-energy, cosine-law emission in all directions) than for backscattered electrons (higher-energy, more specular reemission). This seems to produce a shift to lower energies of the SEY from rough surfaces. In this sense, low-energy ion bombardment can level rough surfaces or create topographical structures in a smooth surface. For instance, although most calculations ${ }^{8}$ seem to indicate a general increase of SEY with surface roughness, one of the methods to reduce secondary emission electrons on copper surfaces is by using an ion-beam process or bake out at atmospheric pressure that produces deeply textured surfaces. ${ }^{9,10}$ In this work, the results on the relationship between the surface nanomorphology and SEY are also shown. Low-energy ion beams were used as a surface-processing tool. Two main types of surface changes of the thin film materials induced by low-energy ion bombardment were studied, namely, modification of the electronic structure and modification of the morphology.

\section{EXPERIMENT}

Amorphous hydrogenated carbon $(a-\mathrm{C}: \mathrm{H})$ of the type called diamondlike carbon (DLC) and fullerene $\left(\mathrm{C}_{60}\right)$ were deposited by $\mathrm{CH}_{n}{ }^{+}$ion bombardment and sublimation of $\mathrm{C}_{60}$ 
powder, respectively, onto (100) silicon wafers in an ultrahigh vacuum chamber, directly attached to the photoemission analyzer. The background pressure was $2 \times 10^{-9}$ mbar. Carbon nitride was synthesized by low-energy $\mathrm{N}_{2}{ }^{+}$ion bombardment at $750 \mathrm{eV}$ in $\mathrm{C}_{60}, a-\mathrm{C}: \mathrm{H}$, and pyrolithic polycrystalline graphite. ${ }^{11,12}$ The ion source was of the Penning cold-cathode type, producing an ion flux of $10 \mu \mathrm{A}$ on a surface of $1.5 \mathrm{~cm}^{2}$ $\left(\sim 8 \times 10^{13}\right.$ ions $\left./ \mathrm{cm}^{2} \mathrm{~s}\right)$. The samples were also bombarded by $\mathrm{Ar}^{+}$ions of $500 \mathrm{eV}$ at a dose of $0.12 \mathrm{C} / \mathrm{cm}^{-2}$.

Ultraviolet photoemission spectroscopy (UPS) was performed at the TGM2 beam line of the BESSY synchrotron of Berlin (Germany) using a VG ADES 400 system with a hemispherical energy analyzer with $4^{\circ}$ acceptance angle. Valence band (VB) photoemission spectra in the $30-172 \mathrm{eV}$ photon energy range were recorded. Combined energy resolution of the monochromator and the analyzer was found to be better than $0.4 \mathrm{eV}$. X-ray photoelectron spectroscopy (XPS) was performed in a VGS ESCALAB 210 instrument using a nonmonochromatic $\mathrm{Mg} K \alpha$ x-ray source $(h \nu$ $=1253.6 \mathrm{eV}$ ). The combined resolution of the analyzer and the $K \alpha 1,2$ line was $0.75 \mathrm{eV}$. The total secondary electron emission coefficient $\sigma$, being $\sigma=\delta+\eta$, with $\delta$ as the true secondary electron yield and $\eta$ as the backscattered electrons per primary electron, was measured in situ as a function of the primary electron beam energy $E_{p}$ from 0 to $2000 \mathrm{eV}$ by measuring the sample current to ground $I_{s}$ while the electron beam was impinging normally on the sample surface. The sample was biased at $-27 \mathrm{eV}$ to repel secondary electrons. We have calculated the total secondary electron emission coefficient from the ratio $\sigma\left(E_{p}\right)=1-I_{s} / I_{p}$, where $I_{p}$ is the primary current of the electron gun. $I_{p}$ was obtained by applying a relative calibration method using the total secondary electron emission coefficient of platinum as a reference.

Atomic force microscope (AFM) measurements were performed with a Nanoscope IIIa equipment (from Digital Instruments, CA) at ambient conditions. Images were taken in both contact and tapping modes. For the contact mode, silicon nitride cantilevers were employed whereas for the tapping mode silicon cantilevers were used. Multipactor tests were performed at ESA/ESTEC test facilities using a dedicated vacuum chamber. The rf signal was chosen to be at $5.3 \mathrm{GHz}$ using a pulse width of $37 \mu$ s and a duty cycle of $1.7 \%$ with a parallel plate sample geometry with a gap of $2.0 \mathrm{~mm}$ and a plate size of $30.0 \mathrm{~mm}$ length and $15.0 \mathrm{~mm}$ width. The breakdown event was monitored using spectrum analyzers, which are able to detect changes in the harmonic level and in the reflected signal when the discharge takes place. An electron detector and photomultiplier were also used as additional detection methods.

\section{RESULTS AND DISCUSSION}

Figure 1(a) shows the SEY coefficient $\sigma$ versus the primary electron energy $E_{p}$ for $a-\mathrm{C}: \mathrm{H}$ exposed to air before (curve i) and after $\mathrm{Ar}^{+}$ion bombardment (curve ii). Relevant parameters are the maximum values of SEY $\sigma_{m}$ at the energy $E_{m}$, and both crossovers for $\sigma=1, E_{1}$, and $E_{2}$ (marked in curve i). Table I presents the experimental values of these SEY parameters. After $\mathrm{Ar}^{+}$ion bombardment, $\sigma_{m}$ decreases,

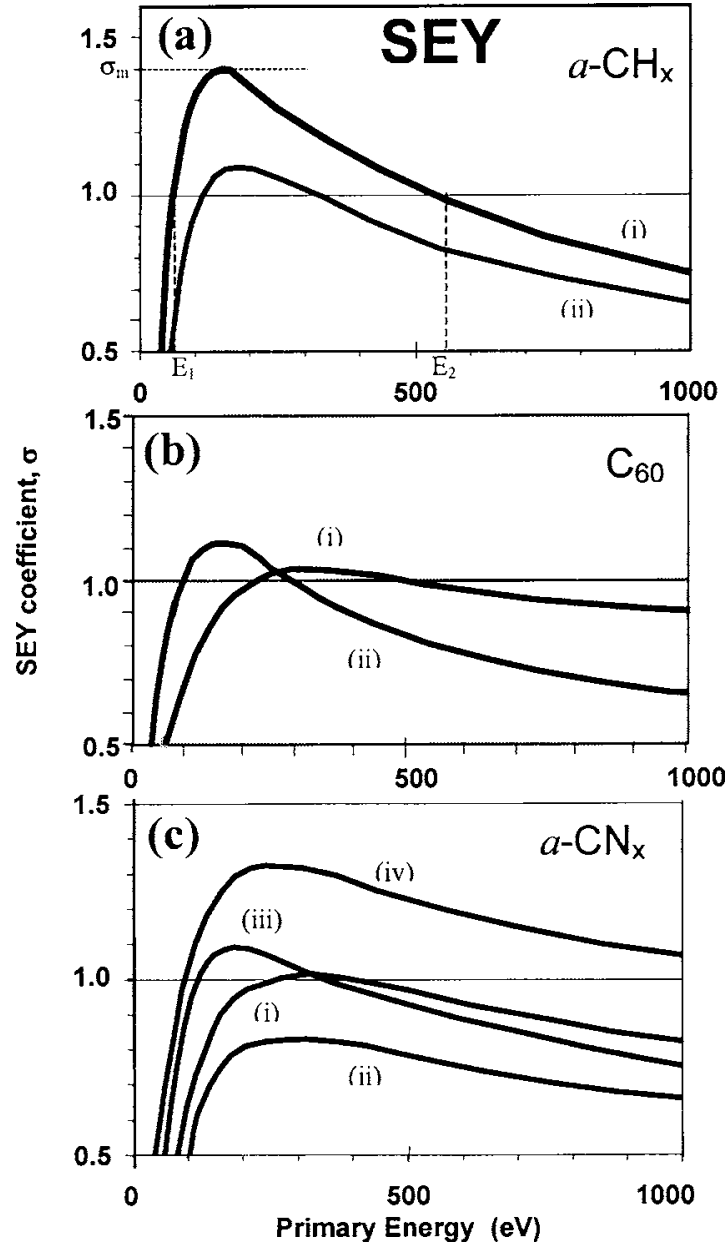

FIG. 1. Total SEY coefficient vs primary electron energy of (a) $a-\mathrm{CH}_{x}$ deposited by $\mathrm{CH}_{4}$ plasma after air exposure (curve i) and after $\mathrm{Ar}^{+}$ion (500 eV and $10^{16} \mathrm{~cm}^{-2}$ ) bombardment (curve ii); (b) $\mathrm{C}_{60}$ exposed to air (curve i) and after $\mathrm{Ar}^{+}$ion bombardment (curve ii); and (c) clean pyrolithic polycrystalline graphite (curve i), after $\mathrm{N}_{2}{ }^{+}(1000-250 \mathrm{eV}$ and 2 $\times 10^{18} \mathrm{~cm}^{-2}$ ) bombardment $\left(a-\mathrm{CN}_{x}\right)$ (curve ii), after 6 days of air exposure (curve iii), and typical high $V_{\text {thres }}$ Alodine (curve iv).

$E_{m}$ and $E_{1}$ shift toward higher primary energies, and $E_{2}$ shifts toward lower primary energies. Figure 1(b) shows the $\sigma\left(E_{p}\right)$ curves of $\mathrm{C}_{60}$ fullerene exposed to air before and after $\mathrm{Ar}^{+}$ ion bombardment of $500 \mathrm{eV}$, curves and ii, respectively. Also, a decrease in $\sigma_{m}$ is observed after $\mathrm{Ar}^{+}$ion bombardment. This decrease is related to the determinant step of the escape through the surface that is affected by low-energy ion bombardment and to the cleaning effect of the surface.

Figure 1(c) (curve i) shows the $\sigma\left(E_{p}\right)$ curve measured for clean pyrolithic polycrystalline graphite. The SEY curves of the amorphous carbon $a$-C (not shown) produced by lowenergy $\mathrm{Ar}^{+}$ion bombardment of graphite are similar than those of pyrolithic polycrystalline graphite. However, $a-\mathrm{C}: \mathrm{H}$ has a high relative $\sigma_{m}$ [Fig. 1(a) (curve i)] as compared with graphite that can be attributed both to its diamondlike bonding $\left(s p^{3}\right)$ and hydrogen content [in this case 30 at. \% as we measured by elastic recoil detection analysis (ERDA)]. This could be explained by the formation of an energy band gap and surface states that help secondary electrons to diffuse and tunnel through the surface energy barrier. The presence of an energy gap can prevent low-energy sec- 
TABLE I. Comparison of the SEY parameters of $a-\mathrm{C}: \mathrm{H}, \mathrm{C}_{60}$, and graphite before and after low-energy ion bombardment.

\begin{tabular}{|c|c|c|c|c|c|c|c|}
\hline Sample & $\sigma_{m}$ & $\begin{array}{c}E_{m} \\
(\mathrm{eV})\end{array}$ & $\begin{array}{c}E_{1} \\
(\mathrm{eV})\end{array}$ & $\begin{array}{c}E_{2} \\
(\mathrm{eV})\end{array}$ & $\begin{array}{c}\mathrm{Ar}^{+} \text {ion } \\
\text { bombardment }{ }^{\mathrm{a}}\end{array}$ & $\begin{array}{c}\mathrm{N}_{2}^{+} \text {ion } \\
\text { bombardment }^{\mathrm{b}}\end{array}$ & $\underset{\text { exposure }^{c}}{\text { Air }}$ \\
\hline$a-\mathrm{C}: \mathrm{H}^{\mathrm{d}}$ & 1.40 & 156 & 70 & 535 & $\cdots$ & $\cdots$ & yes \\
\hline$a-\mathrm{C}: \mathrm{H}^{\mathrm{e}}$ & 1.10 & 200 & 112 & 327 & yes & $\cdots$ & $\ldots$ \\
\hline $\mathrm{C}_{60}{ }^{\mathrm{f}}$ & 1.12 & 160 & 100 & 310 & $\cdots$ & $\cdots$ & yes \\
\hline $\mathrm{C}_{60}{ }^{\mathrm{g}}$ & 1.02 & 270 & 200 & 380 & yes & $\cdots$ & $\cdots$ \\
\hline Graphite $^{\mathrm{h}}$ & 1.00 & 290 & $\cdots$ & $\cdots$ & $\cdots$ & $\cdots$ & yes \\
\hline $\begin{array}{l}\text { Nitrided graphite and } \\
\mathrm{C}_{60}{ }^{\mathrm{i}}\end{array}$ & 0.80 & 250 & $\cdots$ & $\cdots$ & $\cdots$ & yes & $\cdots$ \\
\hline $\begin{array}{l}\text { Nitrided graphitte and } \\
\mathrm{C}_{60}{ }^{j}\end{array}$ & 1.08 & 190 & 110 & 330 & $\cdots$ & yes & yes \\
\hline Alodine $^{\mathrm{k}}$ & 3.10 & 220 & 100 & $\ldots$ & $\cdots$ & $\cdots$ & yes \\
\hline
\end{tabular}

\footnotetext{
${ }^{\mathrm{a} A f t e r ~} \mathrm{Ar}^{+}$ion bombardment of $500 \mathrm{eV}$ at a dose of $0.12 \mathrm{C} \mathrm{cm}^{-2}$.

${ }^{\mathrm{b}}$ After $\mathrm{N}_{2}{ }^{+}$ion bombardment of $750 \mathrm{eV}$.

${ }^{\mathrm{c}}$ After 6 days of air exposure.

${ }^{\mathrm{d}}$ Figure 1(a) (curve i).

${ }^{\mathrm{e}}$ Figure 1(a) (curve ii).

${ }^{\mathrm{f}}$ Figure 1(b) (curve i).

${ }^{\mathrm{g}}$ Figure 1(b) (curve ii).

${ }^{\mathrm{h}}$ Figure 1(c) (curve i).

${ }^{\mathrm{i}}$ Figure 1(c) (curve ii).

${ }^{\mathrm{j}}$ Figure 1(c) (curve iii).

${ }^{\mathrm{k}}$ Figure 1(c) (curve iv).
}

ondary electrons from losing energy through electronelectron collisions, thereby resulting in a large diffusion length for the secondary electrons and a large secondary electron yield. In this sense, it is worth mentioning that the secondary electron yield of graphite increases after hydrogen implantation. ${ }^{13}$ Moreover, hydrogen has also been found to shift the conduction band minimum to energies close to, or even above, the vacuum level on diamond surfaces, ${ }^{14,15}$ that is to say, to create a negative electron affinity surface. In all cases, oxygen incorporation in carbon after air exposure (observed by XPS) increases its secondary electron emission.

Figure 1(c) also shows the SEY curves measured for graphite after nitridation (curve ii) and the subsequent air exposure (curve iii). The SEY results of Alodine alloy, originally the standard reference antimultipactor coating used in the aerospace industry, are also included for comparison purposes. In contrast to the effect induced by hydrogen and oxygen incorporations, a marked decrease in $\sigma_{m}$ after the nitrogen incorporation in carbon (graphite, $a-\mathrm{C}: \mathrm{H}$, and $\mathrm{C}_{60}$ ) is produced. $\mathrm{CN}_{x}$ surface $(x=0.35$, result obtained by the XPS analysis) has a value of $\sigma_{m}$ lower than unity and, in consequence, no multipactor would be possible at any rf intensity. In addition, nitrogen bombardment is more efficient for the secondary emission reduction than bombardment by $\mathrm{Ar}^{+}$ions. It is noteworthy that this low $\sigma_{m}$ value had deteriorated to a very minor extent by exposure to air during 6 days (curve iii). Carbon is passivated by bonding to nitrogen and, therefore, does not absorb as much water and oxygen when exposed to air.

We have studied the relationship between the scale of surface roughness obtained by AFM and the secondary electron emission properties of $\mathrm{C}_{60}, a-\mathrm{C}: \mathrm{H}$, and $\mathrm{CN}_{x}$. Some representative results are shown in Fig. 2. The surface of the $\mathrm{C}_{60}$ thin film deposited by evaporation onto a silicon substrate shows an aggregatelike morphology (bunches of grains) with a root mean square roughness of $150 \mathrm{~nm}$. The flatter regions not including the aggregates show an overall roughness of $35 \mathrm{~nm}$ [Fig. 2(a)]. The secondary emission curves $\sigma\left(E_{p}\right)$ [Fig. 1(b)] could be explained by the facts that (i) the pronounced maximum of $\sigma$ for $E_{p} \sim 170 \mathrm{eV}$ can be due to the approximate coincidence of the penetration range of primaries of this energy with both the secondary diffusion length and characteristic length of the surface, and (ii) the low $\sigma$ observed at large $E_{p}$ is due to the low total electron density of the material (the $\mathrm{C}_{60}$ molecule is an open structure and, moreover, the film shows an open morphology). After $\mathrm{N}_{2}{ }^{+}$ ion bombardment, the surface of the $\mathrm{C}_{60}$ thin film is modified to a smoother uniform surface with a roughness of $2 \mathrm{~nm}$ [Fig. 2(b)]. Correspondingly, the maximum $\sigma_{m}$ smoothes out but $\sigma$ increases at large energies (because the fullerene is compacted). Although the AFM images of the $a-\mathrm{CH}_{x}$ [Fig. 2(c)] reveal a very flat surface, the relatively high $\sigma_{m}$ is explained by the presence of hydrogen that increases the secondary electron emission coefficient.

The electron photoemission from surfaces is related to the secondary emission process. The photoelectrons correspond to the elastically reflected and reemitted ones in secondary electron emission process, but photoelectrons carry the information of the binding energy, i.e., of the energy state they come from. Before being emitted to vacuum, the photoelectrons in the material can have energy losses and generate secondary electrons, just as in the secondary electron emission process. Figure 3 presents the photoemission spectra of both graphite and $\mathrm{C}_{60}$ fullerene before and after $\mathrm{N}_{2}{ }^{+}$ ion-beam bombardment. This measurement was performed with photons of $40 \mathrm{eV}$ to ensure the minimum probe depth of two or three atomic layers. The curves i and ii show the UPS for polycrystalline graphite before and after $\mathrm{N}_{2}{ }^{+}$ion bombardment. Spectrum i also shows some structure in the density of states of the valence band due to the hexagonal sym- 

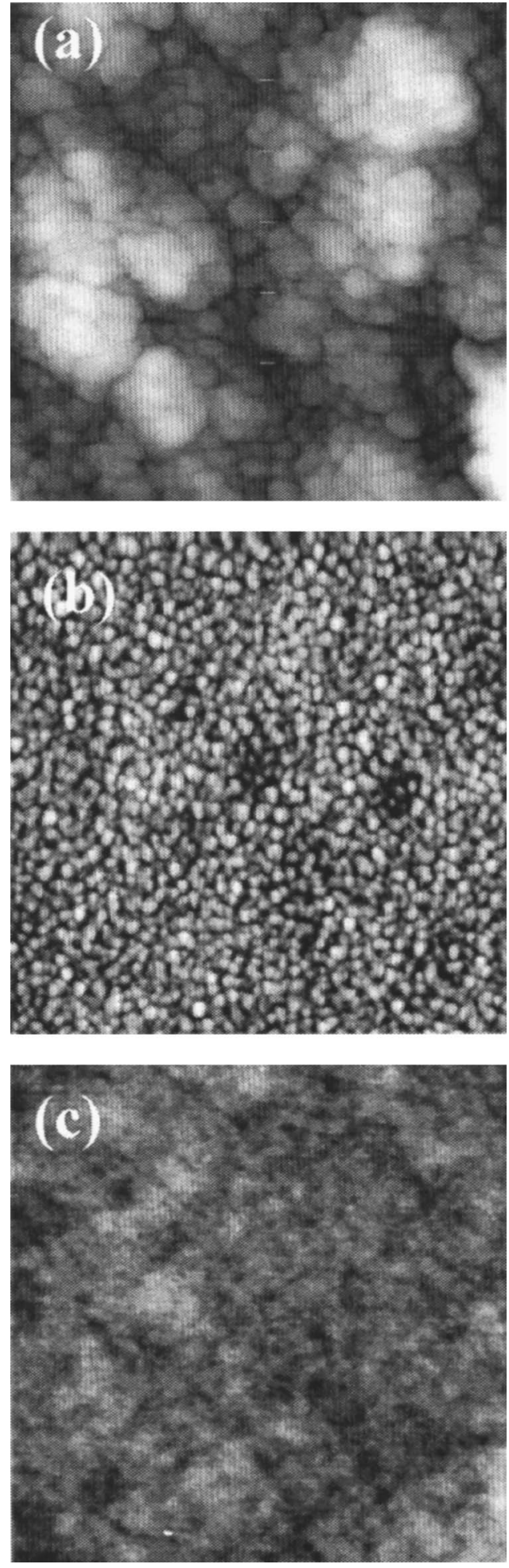

FIG. 2. $6 \times 6 \mu \mathrm{m}^{2} \mathrm{AFM}$ images of (a) $\mathrm{C}_{60}$ fullerene evaporated on single crystal $\mathrm{Si}$, (b) carbon nitride obtained by $\mathrm{N}_{2}{ }^{+}\left(500 \mathrm{eV}\right.$ and $\left.10^{17} \mathrm{~cm}^{-2}\right)$ ion bombardment of $\mathrm{C}_{60}$, and (c) $a-\mathrm{C}: \mathrm{H}$ obtained by $\mathrm{CH}_{4}$ plasma on single crystal.

metry of its crystal structure. This structure is washed out by ion bombardment producing an amorphous material. However, the density of states close to the Fermi level, already high in graphite, remains very similar after bombardment by $\mathrm{N}_{2}{ }^{+}$ions.

The photoemission spectrum of fullerene (curve iii) shows a marked structure in the range of $22-38 \mathrm{eV}$ (kinetic

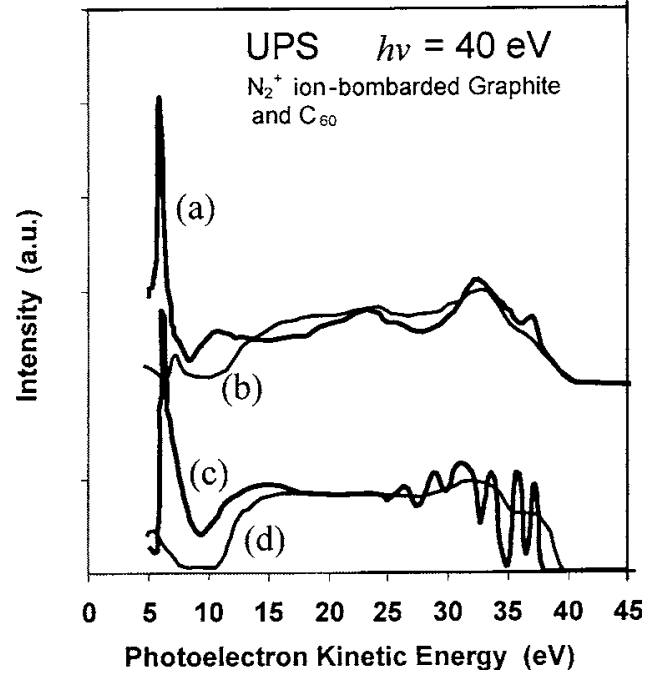

FIG. 3. UPS of $\mathrm{N}_{2}^{+}$ion bombarded pyrolithic polycrystalline graphite and $\mathrm{C}_{60}$ : (a) graphite, (b) last after $\mathrm{N}_{2}^{+}\left(250 \mathrm{eV}\right.$ and $\left.2 \times 10^{17} \mathrm{~cm}^{-2}\right)$ bombardment, (c) $\mathrm{C}_{60}$, and (d) after $\mathrm{N}_{2}{ }^{+}\left(50 \mathrm{eV}\right.$ and $\left.5 \times 10^{15} \mathrm{~cm}^{-2}\right)$ bombardment.

energy measured with respect to the Fermi level) corresponding to the valence levels of the highly symmetric $\mathrm{C}_{60}$ molecule. There are no energy levels just below the Fermi level $(38-40 \mathrm{eV})$. Besides, the information on the escape process can be obtained from the part of the spectrum corresponding to low kinetic energies. No electron is emitted with energy below $5 \mathrm{eV}$, which is the work function of the material. Just above the vacuum level an intense and narrow peak is observed due to secondaries that escape through discrete energy levels of the surface states due to surface defects. After $\mathrm{N}_{2}{ }^{+}$ ion bombardment of $\mathrm{C}_{60}$, this tunneling energy level disappears $^{16}$ (spectrum iv) and, in general, the density of empty states close to the vacuum level decreases significantly. At the other extreme, the nearly discrete valence levels of the $\mathrm{C}_{60}$ valence band become wide solid-state bands extending up to the Fermi level with a density of states similar or greater than that of graphite. The material becomes a relatively good conductor at the surface upon ion bombardment. In a conductor, the diffusion length of secondaries is much smaller than that in an insulator and, therefore, the amount of electrons that can be emitted decreases.

The multipactor threshold power or multipactor breakdown power (MPT) for carbon nitride coatings tested at ESTEC was $7.5 \mathrm{~kW}, 10 \%$ higher than the previously measured on graphite before nitridation. In addition, the enhancement in multipactor threshold power by using $\mathrm{CN}$ instead of the standard antimultipactor coating, Alodine, is 20\%. The relation between secondary electron emission properties and multipactor threshold (or multipactor breakdown voltage) $V_{\text {thres }}$ is more complicated than expected because both measurements cannot be made simultaneously. In addition, the measurement of $V_{\text {thres }}$ itself modifies the SEY of the film. From multipactor threshold tests and computer simulation studies, ${ }^{17,18}$ two parameters of secondary electron emission influencing $V_{\text {thres }}$ appear to be dominant: $\sigma_{m}$ and $E_{1}$. In fact, a rough rule of thumb $\mathrm{MPT} \propto\left(E_{1} / \sigma_{m}\right)^{1 / 2}$ seems to hold for $E_{1} / \sigma_{m} \leqslant 60 \mathrm{eV}$. Thus, in order to minimize or even reduce multipactor effects it would be interesting to increase $V_{\text {thres }}$ 
as much as possible. Therefore, for technical purposes, the value of $E_{1}$ should be maximized whereas $\sigma_{m}$ should be minimized.

In summary, low-energy ion bombardment with $\mathrm{N}_{2}{ }^{+}$of $a-\mathrm{C}: \mathrm{H}$, graphite, and $\mathrm{C}_{60}$ produces $\mathrm{C}-\mathrm{N}$ bonding and reduces their SEY, and also deeply textures the surface to a nanometric scale with the property of hindering secondary electron emission. However, hydrogen or oxygen incorporation into the carbon coatings causes an increase in the SEY of the films. The decrease in secondary electron yield after nitridation of carbon was correlated with the valence band photoemission spectra. These results indicate that $\mathrm{CN}_{x}$ is favorable for low secondary electron emission applications since (a) the resulting material has low total (including core atomic orbitals) electronic density while maintaining a good conductivity similar to that to graphite, and (b) also, it exhibits a zero band gap as indicated by the valence band spectrum.

\section{ACKNOWLEDGMENTS}

We would like to thank Carlos E. Montesano of EADSCASA for his collaboration. This work was supported by the Ministry of Science and Technology of Spain through the coordinated Project Nos. ESP2002-04509-C04-04 and ESP2002-04509-C04-02, and the TMR Project No. ERBFMGECT950022 of the European Community.
${ }^{1}$ R. A. Kishek and Y. Y. Lau, Phys. Rev. Lett. 80, 193 (1998).

${ }^{2}$ P. Yla-Oijala and M. Ukkola, Nucl. Instrum. Methods Phys. Res. A 474, 197 (2001).

${ }^{3}$ D. Wolk et al., Proceedings of the Fourth International Workshop on Multipactor, Corona and Passive Intermodulation in Space RF Hardware: Testing and Hardware Development in RF Breakdown, ESA/ESTEC, Noordwijk, 2003, ITT ESA Report No. AO-4025, 2003.

${ }^{4}$ J. R. M. Vaughan, IEEE Trans. Electron Devices 35, 1172 (1988).

${ }^{5}$ R. A. Kishek, Y. Y. Lau, L. K. Ang, A. Valfells, and R. M. Gilgenbach, Phys. Plasmas 5, 2120 (1998).

${ }^{6}$ F. Höhn, W. Jacob, R. Beckmann, and R. Wilhelm, Phys. Plasmas 4, 940 (1997).

${ }^{7}$ R. Cimino, I. R. Collins, M. A. Furman, M. Pivi, F. Ruggiero, G. Rumolo, and F. Zimmermann, Phys. Rev. Lett. 93, 014801 (2004).

${ }^{8}$ J. Kawata and K. Ohya, J. Phys. Soc. Jpn. 63, 795 (1994)

${ }^{9}$ A. N. Curren, K. A. Jensen, and R. F. Roman, NASA Report No. TP-2967 (1995).

${ }^{10}$ V. Baglin, B. Henrist, N. Hilleret, E. Mercier, and C. Scheuerlein, Proceeding of Chamonix X, Switzerland, 2000, CERN Report No. CERN-SL2000-007 D1, 2000.

${ }^{11}$ J. M. Ripalda et al., Phys. Rev. Lett. 85, 2132 (2000).

${ }^{12}$ I. Montero, L. Galán, J. M. Ripalda, and C. Sánchez Grande, Chem. Phys. Lett. 286, 485 (1998).

${ }^{13}$ K. Nishimura and K. Ohya, Jpn. J. Appl. Phys., Part 1 32, 2856 (1993).

${ }^{14}$ G. T. Mearini, I. L. Krainsky, J. A. Dayton, Jr., Y. Wang, C. A. Zorman, J. C. Angus, and R. W. Hoffman, Appl. Phys. Lett. 65, 2702 (1994).

${ }^{15}$ T. P. Humphreys, R. E. Thomas, D. P. Malta, J. B. Posthill, M. J. Mantini, R. A. Rudder, G. C. Hudson, and R. J. Markunas, Appl. Phys. Lett. 70, 1257 (1997).

${ }^{16}$ J. Halbritter, J. Appl. Phys. 53, 6475 (1982).

${ }^{17}$ L. Galán, M. A. Jiménez, and F. Rueda, ESA-ESTEC Report No. 6577/ 85/NL/PB, 1990 (unpublished).

${ }^{18}$ N. Díaz, S. Castañeda, J. M. Ripalda, I. Montero, L. Galán, F. Feltham, D. Raboso, and F. Rueda, 6th Spacecraft Charging Technology Conference, AFRL-VS-TR-20001578, 1 September 2000. 